Journal of Animal and Veterinary Advances 18 (7): 201-207, 2019

ISSN: 1680-5593

(C) Medwell Journals, 2019

\title{
Nephroprotective Effects of Cinnamon and/or Parsley Oils against Gentamicin-Induced Nephrotoxicity in Rats
}

\author{
${ }^{1}$ Ashraf Elkomy, ${ }^{1}$ Mohamed Aboubakr, ${ }^{1}$ Yara Medhat, ${ }^{2}$ Amira Abugomaa and ${ }^{1}$ Mohamed Elbadawy \\ ${ }^{1}$ Department of Pharmacology, Faculty of Veterinary Medicine, Benha University, \\ 13736 Moshtohor, Toukh, Qalioubeya, Egypt \\ ${ }^{2}$ Faculty of Veterinary Medicine, Mansoura University, 35516 Mansoura, Egypt
}

\begin{abstract}
In the present study, the nephroprotective potentials of cinnamon and/or parsley oils was assessed on gentamicin (GM)-induced nephrotoxicity in rats. About 49 male rats were assigned randomly into 7 equal

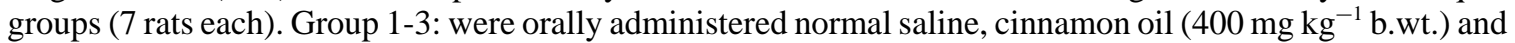
parsley oil (200 $\mathrm{mg} \mathrm{kg}^{-1}$ b.wt.), respectively, once daily for 30 consecutive days. Group 4: rats in this group were intraperitoneally injected with GM (100 $\mathrm{mg} \mathrm{kg}^{-1}$ b.wt.) in the last 10 days (20-30 $0^{\text {th }}$ day) of the experiment. Group 5: rats in this group were given cinnamon and GM while rats in group 6 were administered parsley and GM. Group 7: rats in this group were administered cinnamon and parsley plus GM. At the end of the experiment, rats were sacrificed, serum samples and kidney specimens were collected for biochemical and oxidative status evaluation. Rats administered GM alone showed significant increases in serum concentrations of creatinine, urea and glucose while renal tissue levels of superoxide dismutase, catalase and glutathione reductase were significantly declined. Additionally, the malondialdehyde level was significantly elevated in kidney tissues. Also, GM administration caused renal histopathological alterations. The altered levels of various parameters provoked by GM toxicity were restored towards normal levels by cinnamon and/or parsley oils administration. These results suggested the nephroprotective potentials of cinnamon and/or parsley oils in rats, probably triggered by their antioxidant phytoconstituents.
\end{abstract}

Key words: Cinnamon, gentamicin, oxidative stress, parsley, nephrotoxicity, rats

\section{INTRODUCTION}

The kidney is important in cleaning wastes and toxins from the blood (like creatinine and urea), regulating electrolyte balance, blood pressure and hormone secretions (Wu et al., 2017). Because of its high vascularity and complex metabolic activities, many drugs and environmental xenobiotics intoxicate the kidney (Elsayed et al., 2013, 2014). Nephrotoxicity is an important side effect of several antibiotics that may lead to acute kidney failure, especially in renal insufficiency patients if taken without considerations.

The aminoglycoside antibiotics, especially, gentamicin (GM) are widely prescribed for life-menacing infections caused by Gram-negative bacteria (Randjelovic et al., 2012). Renal proximal tubulesare the main venue of GM excretion and reabsorption and are usually accumulated within it causing renal damage (Ghaznavi et al., 2016) which limits the therapeutic prescribing of GM. This requires decreasing the total dose of GM and duration of treatment, especially in patients with renal insufficiency (Acharya et al., 2013). GM-triggered renal impairment is usually accompanied by the pronounced release of reactive oxygen and nitrogen species (ROS and RNS), superoxide anions, hydrogen peroxide and hydroxyl radicals by kidney mitochondria (Yang et al., 1995; Tavafi and Ahmadvand, 2011).

The plant extracts-derived phyto-medical agents represent a substantial portion of traditional medicine and are increasingly used to treat a wide range of diseases (Gupta et al., 2004). Antioxidants act as free radical scavengers, inhibiting lipid peroxidation and oxidation processes and protect the human body from several diseases attributed to the reactions of radicals (Kurowska and Gaiazzka, 2006; Lee et al., 2010). Therefore, the protective effects of natural herbal-derived antioxidants against drug-provoked side effects need more attention. (Frei and Higdon, 2003). Because of its enrichment by polyphenolic compounds, cinnamon (Cinnamomum zeylanicum) is regarded as a powerful natural antioxidant. In folk medicine, cinnamon is widely used as antioxidant, anti-diabetic, anti-microbial effects and anti-inflammatory herb (Elkomy et al., 2016; Abdeen et al., 2019). The main constituents identified in the cinnamon extract and have powerful free radical scavenging activities are cinnamaldehyde, eugenol and cinnamic acid which mainly restores the redox hemostasis

Corresponding Author: Mohamed Aboubakr, Department of Pharmacology, Faculty of Veterinary Medicine, Benha University, Moshtohor, Toukh, Elqaliobiya, 13736, Egypt, mohamed.aboubakr@fvtm.bu.edu.eg 
and normal cellular function (Hafizur et al., 2015). Parsley (Petroselinum crispum) is mainly utilized as a flavoring agent in food manufacturing or as a fragrance in perfumery and cosmetics factories. Parsley was previously reported to show weak antioxidant, diuretic and antimicrobial activities (Teissedre and Waterhouse, 2000). Moreover, myristicin an important constituent from parsley oil, revealed a potential cancer chemoprotective agent (Benevides et al., 1999). According to our knowledge and literature data, there were no studies concerning cinnamon and parsley combination for protection against nephrotoxicity. Therefore, the purpose of the present study was to check if the renal impairment induced by GM can be ameliorated by cinnamon and/or parsley oils pretreatments in rats.

\section{MATERIALS AND METHODS}

Drugs: Gentamicin sulfate was kindly supplied by Memphis Pharm and Chemical Ind., Cairo, Egypt) and used in the present study to induce nephrotoxicity. Cinnamon and parsley oils were obtained from El-Captain Company for extracting natural oils, herbs and cosmetics, El-Obour City, Cairo, Egypt. Chemical kits were purchased from Biodiagnostic, Chemical Co., Giza, Egypt. All chemicals used were of analytical grade.

Experimental animals: A total of 49 Wister albino male rats weighing 190-220 g were obtained from Laboratory Animal Center, Faculty of Veterinary Medicine, Benha University, Egypt. Rats were housed in polypropylene cages under standard environmental conditions of temperature and humidity and received standard commercial pelleted diet with water ad libitum. The experimental protocol was approved by the Ethics Committee of the Faculty of Veterinary Medicine, Benha University and all efforts were done to minimize pain to rats.

Experimental design: Rats were assigned randomly into 7 equal groups (7 rats each). Group 1-3: were orally administered normal saline, cinnamon oil $\left(400 \mathrm{mg} \mathrm{kg}^{-1}\right.$ b.wt., Abdeen et al., 2019) and parsley oil (200 mg kg-1 b.wt., Abdellatief et al., 2017), respectively, once daily for 30 consecutive days. Group 4: rats in this group were intraperitoneally injected with GM (100 mg kg-1 b.wt., Sadeghi et al., 2015) in the last 10 days (20-30 ${ }^{\text {th }}$ day) of the experiment. Group 5: rats in this group were given cinnamon and GM and rats in group 6 were administered parsley and GM. Group 7: rats in this group were administered cinnamon and parsley plus GM.

Sampling: About $24 \mathrm{~h}$ after the last administration, rats were sacrificed under isoflurane anesthesia and blood samples were collected directly from retroorbital plexus for serum separation intended for the biochemical evaluations. Kidneys tissues were collected immediately after complete necropsy of rats, cut into specimens and were washed with a cooled phosphate buffer saline $\left(\mathrm{pH}\right.$ 7.4). Some specimens were kept at $-80^{\circ} \mathrm{C}$ for oxidative cascade determination and the others were fixed immediately in $4 \%$ paraformaldehyde phosphate buffer solution (Wako Pure Chemicals Co., Osaka, Japan) and kept in the refrigerator for histopathological assessments.

Serum biochemical analysis: Serum samples were used for spectrophotometric estimation of creatinine, urea and glucose levels using commercial assay kits (Biodiagnostic, Chemical Co., Giza, Egypt). The serum samples were used for the quantitative assay of creatinine (Bartels et al., 1972), urea (Chaney and Marbach, 1962) and glucose (Sugiura and Hirano, 1977) levels.

Preparation of kidney homogenates and determination of oxidative cascade: About $1 \mathrm{~g}$ of the frozen kidney specimens was allowed to thaw and washed by ice-cold $0.9 \% \mathrm{NaCl}$ solution and then homogenized in $9 \mathrm{~mL}$ ice-cold Phosphate Buffer Saline (PBS, 50 mM, pH: 7.4) using homogenizer. The obtained homogenate was centrifuged at $600 \mathrm{~g}$ for $15 \mathrm{~min}$ at $4^{\circ} \mathrm{C}$ and the supernatant was collected into and kept at $-80^{\circ} \mathrm{C}$ until analysis. The oxidative status evaluation was done by determination of Malondialdehyde (MDA) level (Uchiyama and Mihara, 1978), Catalase (CAT) activity (Aebi, 1984), Superoxide Dismutase (SOD) activity (Nishikimi et al., 1972) and reduced Glutathione (GSH) concentration (Habig et al., 1974).

Histopathological examinations: The formalin-fixed specimens of the kidney were washed, dehydrated, paraffin-embedded, sectioned into three um sections. Thereafter, the sections were deparaffinized and stained with Harris hematoxylin and eosin for general histological examination (Bancroft and Gamble, 2008).

Statistical analysis: Statistical assessment was performed using SPSS (Version 20.0; SPSS Inc., Chicago, IL, USA). The significant differences between groups were evaluated by one-way ANOVA using the Duncan test as a post hoc. Results are expressed as mean \pm SEM. All values at $\mathrm{p} \leq 0.05$ were considered statistically significant.

\section{RESULTS AND DISCUSSION}

Concerning biochemical parameters results, rats administrated GM showed significant $(\mathrm{p}<0.05)$ elevation of creatinine, urea and glucose levels in serum when compared to the control group. Considerable improvement in these parameters was observed following cinnamon and/or parsley oil administration and these results were recorded in Table 1. 
Table 1: Ameliorative effects of orally administered cinnamon oil (400 mg kg- ${ }^{1}$ b.wt.) or parsley oil (20 $\mathrm{mg} \mathrm{kg}^{-1}$ b.wt.) or their combination on some serum renal biochemical changes induced by i.p. injection of gentamicin (100 $\mathrm{mg} \mathrm{kg}^{-1}$ b.wt.) in rats (mean \pm S.E.M, $\mathrm{n}=7$ )

\begin{tabular}{llll}
\hline Groups & Creatinine (mg/dl) & Urea (mg/dl) & Glucose (mg/dl) \\
\hline Control & $0.71 \pm 0.02^{\mathrm{d}}$ & $32.30 \pm 1.34^{\mathrm{d}}$ & $80.21 \pm 3.87^{\mathrm{d}}$ \\
CN & $0.72 \pm 0.01^{\mathrm{d}}$ & $31.61 \pm 1.28^{\mathrm{d}}$ & $79.31 \pm 2.70^{\mathrm{d}}$ \\
PR & $0.69 \pm 0.009^{\mathrm{d}}$ & $29.88 \pm 1.47^{\mathrm{d}}$ & $76.90 \pm 3.44^{\mathrm{d}}$ \\
GM & $1.43 \pm 0.03^{\mathrm{a}}$ & $83.31 \pm 1.54^{\mathrm{a}}$ & $193.53 \pm 2.41^{\mathrm{a}}$ \\
GM+CN & $1.10 \pm 0.02^{\mathrm{b}}$ & $71.70 \pm 2.24^{\mathrm{b}}$ & $157.15 \pm 1.34^{\mathrm{b}}$ \\
GM+PR & $1.06 \pm 0.01^{\mathrm{b}}$ & $73.08 \pm 1.94^{\mathrm{b}}$ & $153.87 \pm 2.19^{\mathrm{b}}$ \\
GM+CN+PR & $0.89 \pm 0.01^{\mathrm{c}}$ & $58.85 \pm 1.27^{\mathrm{c}}$ & $101.84 \pm 2.18^{\mathrm{c}}$ \\
\hline
\end{tabular}

Means within the same column carrying different superscripts are significant at $(\mathrm{p}<0.05)$. CN; Cinnamon, PR; Parsley, GM; Gentamicin

Table 2: Ameliorative effects of orally administered cinnamon oil (400 mg $\mathrm{kg}^{-1}$ b.wt.) or parsley oil (20 mg kg- ${ }^{1}$ b.wt.) or their combination on renal tissues levels of oxidative stress biomarkers induced by i.p. injection of gentamicin $(100 \mathrm{mg} / \mathrm{kg}$ b.wt.) in rats (mean \pm S.E.M, $n=7$ )

\begin{tabular}{lllll}
\hline Groups & MDA(nmol/g) & CAT(U/g) & SOD(U/g) & GSH(mg/g) \\
\hline Control & $58.81 \pm 0.99^{\mathrm{de}}$ & $3.55 \pm 0.06^{\mathrm{a}}$ & $25.57 \pm 0.63^{\mathrm{a}}$ & $60.86 \pm 0.76^{\mathrm{a}}$ \\
CN & $59.92 \pm 2.25^{\mathrm{de}}$ & $3.62 \pm 0.04^{\mathrm{a}}$ & $25.16 \pm 0.30^{\mathrm{a}}$ & $59.19 \pm 1.10^{\mathrm{a}}$ \\
PR & $55.05 \pm 2.74^{\mathrm{e}}$ & $3.59 \pm 0.04^{\mathrm{a}}$ & $23.98 \pm 0.51^{\mathrm{b}}$ & $58.42 \pm 1.93^{\mathrm{a}}$ \\
GM & $122.78 \pm 3.67^{\mathrm{a}}$ & $1.75 \pm 0.04^{\mathrm{d}}$ & $11.67 \pm 0.31^{\mathrm{e}}$ & $31.16 \pm 0.17^{\mathrm{d}}$ \\
GM+CN & $98.73 \pm 3.14^{\mathrm{b}}$ & $2.40 \pm 0.02^{\mathrm{c}}$ & $15.06 \pm 0.29^{\mathrm{d}}$ & $40.75 \pm 0.44^{\mathrm{c}}$ \\
GM+PR & $89.24 \pm 1.53^{\mathrm{c}}$ & $2.36 \pm 0.03^{\mathrm{c}}$ & $14.84 \pm 0.35^{\mathrm{d}}$ & $39.51 \pm 0.68^{\mathrm{c}}$ \\
GM+CN+PR & $65.11 \pm 0.89^{\mathrm{d}}$ & $2.98 \pm 0.02^{\mathrm{b}}$ & $20.30 \pm 0.28^{\mathrm{c}}$ & $49.83 \pm 0.58^{\mathrm{b}}$ \\
\hline
\end{tabular}

Means within the same column carrying different superscripts are significant at $(\mathrm{p}<0.05)$. CN; Cinnamon, PR; Parsley, GM; Gentamicin

Regarding the results of oxidative stress (Table 2), the rats administrated GM showed a marked rise in MDA level and significant decline in CAT, GSH and SOD in renal tissues compared with control groups. Considerable improvement in these parameters was observed following cinnamon and/or parsley administration.

Histopathological examination of renal tissues in different treated groups revealed alterations in their histological architecture. An improvement in histopathology was observed following cinnamon and/or parsley administration (Fig. 1).

The current study was designed to examine the nephroprotective potentials of cinnamon and/or parsley against GM-induced nephrotoxicity in rats, probably due to their antioxidant properties. Kidney damage by antibiotics depends mainly on the dose and the course of the treatment (Benett et al., 1991). Expanded information concerning the side effects of antibiotics will be of benefits to both physicians and their patients (Elsayed et al., 2013, 2014). Gentamicin, the mostly used aminoglycoside antibiotic used at different doses to induce nephrotoxicity, followed in the current study at $100 \mathrm{mg} \mathrm{kg}{ }^{-1}$ b.wt./day to generate nephrotoxicity (Sadeghi et al., 2015).

Determination of serum creatinine level is considered as an important index of renal function (Howard, 1989). Creatinine, a by-product of muscle metabolism and actively secreted by the proximal tubular cells, is excreted unchanged by the kidneys. Its level rises in the blood if there is a shortage in kidney filtration capacity, suggesting remarkable damage to the nephron. Our findings on serum creatinine levels support the existing literature data which showed that GM toxicity is associated with increased serum creatinine concentration (Pedraza-Chaverrı et al., 2000; Maldonado et al., 2003). These further buttresses the fact that GM administration has nephrotoxic potentials.

Urea, produced by the liver in the urea cycle as a waste product of metabolism of protein (either from the oxidation of amino acids or from ammonia) is dissolved into the blood and transported and excreted by the kidney as a component of urine. It is a sensitive biomarker used in the assessment of established renal tissue damage. Therefore, in renal tissue injury, there is retention of urea. Increase urea level is associated with nephritis, renal ischemia, urinary tract obstruction and extra-renal diseases (Oyewole, 2011). The gentamicin-induced increase in serum urea level as observed in the present study is consistent with the findings of Pedraza-Chaverrı et al. (2000) and Maldonado et al. (2003). It has been established that gentamicin causes inhibition of protein synthesis in renal cells with a consequent abundance of amino acid in the kidney resulting in increased urea levels (Sundin et al., 1997). However, cinnamon and/or parsley oils administration significantly reversed the deleterious alteration of plasma total protein levels.

The levels of creatinine and urea in the serum of the groups that received GM and pre-treated with cinnamon and/or parsley oils were significantly declined, suggesting the protective potentials of cinnamon and parsley against kidney damage induced by GM. As mentioned before, after GM treatment, the use of compounds that possesses antioxidant effect improves renal function (Mestry et al., 2018). Serum creatinine and urea levels and the results of pathohistological analysis reflected the development of kidney impairment in GM-administered rats.

In renal tubules, glucose is almost reabsorbed but descend in the urine when their plasma level exceeds the renal threshold and/or due to defect in renal tubules as a result of kidney damage (Elsayed et al., 2013, 2014). The administration of GM caused significant hyperglycemia in the present study. This could be explained based on the lack of glucose filtration due to renal tubular damage induced by GM. This is shown histopathologically in the present study by vacuolization of the lining endothelium of the glomerular tufts with dysplasia and degeneration in the epithelial cells lining of renal tubules (Fig. 1d) with focal inflammatory cells infiltration (Fig. 1d).

It is known that oxidative stress has a pivotal role in the establishment of gentamicin-provoked nephrotoxicity (Walker et al., 1999). Overproduction of ROS alter the oxidant-antioxidant scale and disrupt the integrity of membrane lipid through lipid peroxidation and subsequently increase the MDA, a final metabolite product of lipid peroxidation (Witko-Sarsat et al., 2003; Bekheet et al., 2013; Sahu et al., 2014; 

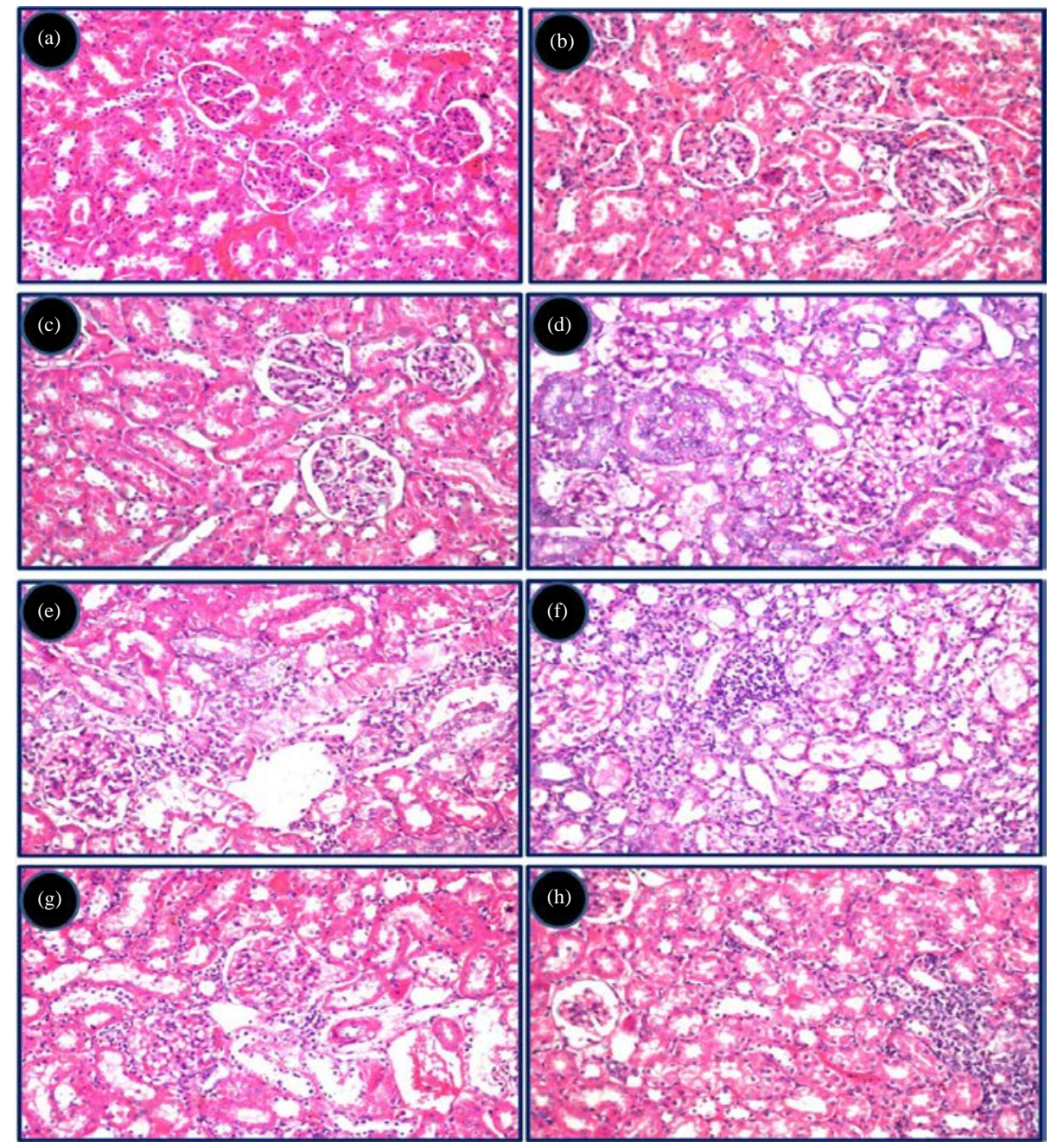

Fig. 1(a-h): Improvement of the renal histopathological changes induced by GM by administration of cinnamon and/or parsley oils to rats. Rat's kidney of the control group (a), cinnamon oil- (b) and parsley oil- (c) treated groups showed the normal histological architecture of the glomeruli and renal tubules. Rat's kidney of gentamicin-treated group showed vacuolization of the lining endothelium of the glomerular tufts with dysplasia in the epithelial cells lining of some tubules (d) and focal inflammatory cells infiltration was detected in between the degenerated tubules at the cortex (e). Rat's kidney of gentamicin-and cinnamon oil-treated group showed focal inflammatory cells aggregation in between the degenerated and necrosed tubules in medullary portion (f). Rat's kidney of gentamicin-and parsley oil-treated group showed focal inflammatory cell infiltration was detected in between the glomeruli and degenerated tubules at the cortex (g). Rat's kidney of gentamicin-cinnamon-and parsley oil-treated group showed focal inflammatory cells infiltration in between the tubules and glomeruli (h)

Devkar et al., 2015). GSH is one of the main intracellular defense systems against oxidative damage via scavenging of hydroxyl radicals and singlet oxygen (Priyamvada et al., 2008; Gosavi et al., 2012; Honmore et al., 2016). SOD is an intracellular antioxidant enzyme which rapidly and specifically reduces superoxide to hydrogen peroxide, a reaction with a 10000 -fold faster rate than spontaneous dismutation (Abdel-Raheem et al., 2009; Lee et al., 2010). CAT, another endogenous antioxidant, detoxify hydrogen peroxide to water (Quiros et al., 2016). For evaluating the antioxidant activities of cinnamon and parsley in renal tissue, we measured the renal GSH level, SOD and CAT activity.

Several studies reported that GM-decreased activity of GSH might be due to excess output of free radicals or increased consumption in the protection of $\mathrm{SH}$ group-containing proteins and decreased SOD antioxidant enzymes accompanied with over output of superoxide 
anions and hydrogen peroxide (Visnagri et al., 2012; Kandhare et al., 2013). In the present study, our results clearly demonstrate that GM caused oxidative renal failure as shown by significant elevation of MDA concentration and significant decline of CAT, SOD and GSH activities in rat's kidney specimens. These oxidative parameters were probably increased due to the reduction of CAT. Similarly, GM administration elevated the MDA level and decreased GSH and SOD activities in renal tissue (Samarghandian et al., 2015). Interestingly, the present findings revealed that the pre-treatment with cinnamon and/or parsley oils significantly restored the MDA level and GSH, CAT and SOD activities in the renal tissue of GM-treated rats (Table 2) via its free radical scavenging and/or antioxidant properties. Our results agree with the previous reports that showed a decline in renal antioxidant enzymes activities, CAT, SOD and GSH in animals treated by GM (Abdel-Naim et al., 1999; Ghaznavi et al., 2016).

In line with the results of the biochemical assessment, histopathological findings demonstrated structural changes in the renal tissue of GM-treated rats. We found that GM administration causes histopathological lesions in kidney, like glomerular atrophy, cell fragments in the tubules and swollen epithelial cells in proximal and distal convoluted tubules. Histopathological results are in rapport with the previous studies (Kalantari et al., 2011). In addition, the protective effects of cinnamon and/or parsley oils were confirmed by histopathological studies of kidney which indicates considerable improvement in proximal and distal convoluted tubules and glomerular atrophy in pre-treated groups (Fig. 1g and h).

\section{CONCLUSION}

We clearly demonstrated that pretreatment with cinnamon and/or parsley oils to GM-treated rats significantly decreased serum creatinine and urea and efficiently lowered renal MDA level compared with GM-treated rats. It also increased SOD, GSH and CAT activities in kidney tissues compared with GM-treated rats. Cinnamon and/or parsley oils treatment mitigated renal damage associated with GM treatment, probably attributed to their potent antioxidant activities and its ability to protect the cell membrane integrity and prevent inflammation. Further, studies are required to elucidate the molecular mechanisms of their protective levels.

\section{REFERENCES}

Abdeen, A., A. Abdelkader, M. Abdo, G. Wareth, M. Aboubakr, L. Aleya and M. Abdel-Daim, 2019. Protective effect of cinnamon against acetaminophen-mediated cellular damage and apoptosis in renal tissue. Environ. Sci. Pollut. Res., 26: $240-249$.
Abdel-Naim, A.B., M.H. Abdel-Wahab and F.F. Attia, 1999. Protective effects of vitamin $E$ and probucol against GM-induced nephrotoxicity in rats. Pharmacol. Res., 40: 183-187.

Abdel-Raheem, I.T., A.A. Abdel-Ghany and G.A. Mohamed, 2009. Protective effect of quercetin against gentamicin-induced nephrotoxicity in rats. Biol. Pharmaceut. Bull., 32: 61-67.

Abdellatief, S.A., A.A.A. Galal, S.M. Farouk and M.M. Abdel-Daim, 2017. Ameliorative effect of parsley oil on cisplatin-induced hepato-cardiotoxicity: A biochemical, histopathological and immunohistochemical study. Biomed. Pharm., 86: 482-491.

Acharya, C.R., H.N. Thakar and S.K. Vajpeyee, 2013. A study of oxidative stress in gentamicin induced nephrotoxicity and effect of antioxidant vitamin C in Wistar rats. National J. Physiol. Pharm. Pharmacol., 3: $14-20$.

Aebi, H., 1984. Catalase in vitro. Meth. Enzymol., 105: 121-126.

Bancroft, J.D. and M. Gamble, 2008. Theory and Practice of Histopathological Techniques. 6th Edn., Churchill Livingstone, New York, London, Madrid.

Bartels, H., M. Bohmer and C. Heierli, 1972. [Serum creatinine determination without protein precipitation]. Clinica Chimica Acta, 37: 193-197, (In German).

Bekheet, S.H., E.A. Awadalla, M.M. Salman and M.K. Hassan, 2013. Prevention of hepatic and renal toxicity with bradykinin potentiating factor (BPF) isolated from Egyptian scorpion venom (Buthus occitanus) in gentamicin treated rats. Tissue Cell, 45: 89-94.

Benevides, P.J., P. Sartorelli and M.J. Kato, 1999. Phenylpropanoids and neolignans from Piper regnellii. Phytochemistry, 52: 339-343.

Bennett, W.M., L.W. Elzinga and G.A. Porter, 1991. Tubulointerstitial Disease and Toxic Nephropathy. In: The Kidney, Brenner, B.M. and F.C. Rector (Eds.). WB Saunders Company, Philadelphia, Pennsylvania, pp: 1430-1496.

Chaney, A.L. and E.P. Marbach, 1962. Modified reagents for determination of urea and ammonia. Clin. Chem., 8: $130-132$.

Devkar, S.T., A.D. Kandhare, B.D. Sloley, S.D. Jagtap and J. Lin et al., 2015. Evaluation of the bioavailability of major withanolides of Withania somnifera using an in vitro absorption model system. J. Adv. Pharmaceut. Technol. Res., 6: 159-164.

Elkomy, A., M. Aboubakr, A. Soliman, A. Abdeen, A. Abdelkader and H. Hekal, 2016. Paracetamol induced hepatic toxicity and amelioration by cinnamon in rats. Int. J. Pharmacol. Toxicol., 4: 187-190. 
Elsayed, M.G.A., A.A.A. Elkomy, M.S. Gaballah and M. Elbadawy, 2014. Nephrotoxicity of cefepime: A new cephalosporin antibiotic in rats. J. Pharmacol. Pharmacother., 5: 33-38.

Elsayed, M.G.A., Elkomy, A.A.A. and M. Elbadawy, 2013. Some pharmacodynamic aspects of cefepime. J. Pharm., Vol. 2013,

Frei, B. and J.V. Higdon, 2003. Antioxidant activity of tea polyphenols in vivo: Evidence from animal studies. J. Nutr., 133: 3275S-3284S.

Ghaznavi, H., S. Mehrzadi, B. Dormanesh, S.M.T.H. Tabatabaei and H. Vahedi, et al., 2016. Comparison of the protective effects of melatonin and silymarin against gentamicin-induced nephrotoxicity in rats. J. Evidence-Based Complementary Altern. Med., 21: NP49-NP55.

Gosavi, T.P., A.D. Kandhare, P. Ghosh and S.L. Bodhankar, 2012. Anticonvulsant activity of Argentum metallicum, a homeopathic preparation. Der Pharmacia Lettre, 4: 626-637.

Gupta, M., U.K. Mazumder, T.S. Kumar, P. Gomathi and R.S. Kumar, 2004. Antioxidant and hepatoprotective effects of Buhinia racemosa against paracetamol and carbon tetrachloride induced liver damage in rats. Iran. J. Pharmacol. Therapeut., 3: 12-20.

Habig, W.H., M.J. Pabst and W.B. Jakoby, 1974. Glutathione S-transferases: The first enzymatic step in mercapturic acid formation. J. Biol. Chem., 249: 7130-7139.

Hafizur, R. M., A. Hameed, M. Shukrana, S.A. Raza, S. Chishti, N. Kabir and R.A. Siddiqui, 2015. Cinnamic acid exerts anti-diabetic activity by improving glucose tolerance in vivo and by stimulating insulin secretion in vitro. Phytomedicine, 22: 297-300.

Honmore, V.S., A.D. Kandhare, P.P. Kadam, V.M. Khedkar and D. Sarkar et al., 2016. Isolates of Alpinia officinarum Hance as COX-2 inhibitors: Evidence from anti-inflammatory, antioxidant and molecular docking studies. Int. Immunopharmacol., 33: 8-17.

Howard, T.E., 1989. Clinical Chemistry. 4th Edn., Wiley Publisher, Hoboken, New Jersey, USA., pp: 58-62.

Kalantari, H., M. Jalali, A. Jalali, A. Salimi and F. Alhalvachi, et al., 2011. Protective effect of Cassia fistula fruit extract on bromobenzene-induced nephrotoxicity in mice. Hum. Exp. Toxicol., 30: 1710-1715.

Kandhare, A.D., S.L. Bodhankar, V. Singh, V. Mohan and P.A. Thakurdesai, 2013. Anti-asthmatic effects of type-A procyanidine polyphenols from cinnamon bark in ovalbumin-induced airway hyperresponsiveness in laboratory animals. Biomed. Aging Pathol., 3: 23-30.
Kurowska, A. and I. Galazka, 2006. Essential oil composition of the parsley seed of cultivars marketed in Poland. Flavour Fragrance J., 21: 143-147.

Lee, W.C., H.C. Chen, C.Y. Wang, P.Y. Lin and T.T. Ou, et al., 2010. Cilostazol ameliorates nephropathy in type 1 diabetic rats involving improvement in oxidative stress and regulation of TGF-ß and NF-?B. Biosci. Biotechnol. Biochem., 74: 1355-1361.

Maldonado, P.D., D. Barrera, I. Rivero, R. Mata, O.N. Medina-Campos, R. Hernandez-Pando and J. Pedraza-Chaverri, 2003. Antioxidant S-allylcysteine prevents gentamicin-induced oxidative stress and renal damage. Free Radic. Biol. Med., 35: 317-324.

Mamoru, S. and H. Kazuyuki, 1977. A new colorimetric method for determination of serum glucose. Clin. Chim. Acta, 75: 387-391.

Mestry, S.N., N.B. Gawali, S.A. Pai, M.S. Gursahani, J.B. Dhodi, R. Munshi and A.R. Juvekar, 2018. Punica granatum improves renal function in gentamicin-induced nephropathy in rats via attenuation of oxidative stress. J. Ayurveda Integr. Med., 16: 30323-30326.

Nishikimi, M., N.A. Rao and K. Yagi, 1972. The occurrence of superoxide anion in the reaction of reduced phenazine methosulfate and molecular oxygen. Biochem. Biophys. Res. Commun., 46: 849-854.

Oyewole, O.I., 2011. Chemopreventive role of vitamin $\mathrm{C}$ and $\mathrm{E}$ on Potassium bromate induced renal oxidative damage in rat. J. Med. Med. Sci., 2: 1189-1192.

Pedraza-Chaverri, J., P.D. Maldonado, O.N. Mediana-Campos, I.M. Olivares-Corichi, M.A. Granados-Silvestre, R. Hernandez-Pando and M.E. Ibarra-Rubio, 2000. Garlic ameliorates gentamicin nephrotoxicity: Relation to antioxidant enzymes. Free Radic. Biol. Med., 29: 602-611.

Priyamvada, S., M. Priyadarshini, N.A. Arivarasu, N. Farooq and S. Khan et al., 2008. Studies on the protective effect of dietary fish oil on gentamicin-induced nephrotoxicity and oxidative damage in rat kidney. Prostaglandins Leukot. Essent. Fatty Acids, 78: 369-381.

Quiros, Y., V. Blanco-Gozalo, J.I. Sanchez-Gallego, F.J. Lopez-Hernandez, J. Ruiz, M.P.P. de Obanos and J.M. Lopez-Novoa, 2016. Cardiotrophin-1 therapy prevents gentamicin-induced nephrotoxicity in rats. Pharmacol. Res., 107: 137-146.

Randjelovic, P., S. Veljkovic, N. Stojiljkovic, L. Velickovic, D. Sokolovic, M. Stoiljkovic and I. Ilic, 2012. Protective effect of selenium on gentamicin-induced oxidative stress and nephrotoxicity in rats. Drug Chem. Toxicol., 35: 141-148. 
Sadeghi, F., M. Nematbakhsh, A. Noori-Diziche, F. Eshraghi-Jazi and A. Talebi et al., 2015. Protective effect of pomegranate flower extract against gentamicin-induced renal toxicity in male rats. J. Renal Inj. Prev., 4: 45-50.

Sahu, B.D., S. Tatireddy, M. Koneru, R.M. Borkar and J.M. Kumar et al., 2014. Naringin ameliorates gentamicin-induced nephrotoxicity and associated mitochondrial dysfunction, apoptosis and inflammation in rats: Possible mechanism of nephroprotection. Toxicol. Applied Pharmacol., 277: 8-20.

Samarghandian, S., M. Azimi-Nezhad, H. Mehrad-Majd and S.R. Mirhafez, 2015. Thymoquinone ameliorates acute renal failure in gentamicin-treated adult male rats. Pharmacol., 96: 112-117.

Sundin, D.P., C. Meyer, R. Dahl, A. Geerdes, R. Sandoval and B.A. Molitoris, 1997. Cellular mechanism of aminoglycoside tolerance in long-term gentamicin treatment. Am. J. Physiol., 272: C1309-C1318.

Tavafi, M. and H. Ahmadvand, 2011. Effect of Rosmarinic acid on inhibition of gentamaicin induced nephrotoxicity in rat. Tissue Cell, 14: 392-397.

Teissedre, P.L. and A.L. Waterhouse, 2000. Inhibition of oxidation of human low-density lipoproteins by phenolic substances in different essential oils varieties. J. Agric. Food Chem., 48: 3801-3805.
Uchiyama, M. and M. Mihara, 1978. Determination of malonaldehyde precursor in tissues by thiobarbituric acid test. Anal. Biochem., 86: 271-278.

Visnagri, A., A.D. Kandhare, V.S. Kumar, A.R. Rajmane and A. Mohammad et al., 2012. Elucidation of ameliorative effect of co-enzyme Q10 in streptozotocin-induced diabetic neuropathic perturbation by modulation of electrophysiological, biochemical and behavioral markers. Biomed. Aging Pathol., 2: 157-172.

Walker, P.D., Y. Barri and S.V. Shah, 1999. Oxidant mechanisms in gentamicin nephrotoxicity. Renal Fail., 21: 433-442.

Witko-Sarsat, V., V. Gausson, A.T. Nguyen, M. Touam, T. Drueke, F. Santangelo and B. Descamps-Latscha, 2003. AOPP-induced activation of human neutrophil and monocyte oxidative metabolism: A potential target for $\mathrm{N}$-acetylcysteine treatment in dialysis patients. Kidney Int., 64: 82-91.

Wu, J., X. Pan, H. Fu, Y. Zheng and Y. Dai et al., 2017. Effect of Curcumin on glycerol-induced acute kidney injury in rats. Sci. Rep., Vol. 7, No. 1. 10.1038/s41598-017-10693-4

Yang, C.L., X.H. Du and Y.X. Han, 1995. Renal cortical mitochondria are the source of oxygen free radicals enhanced by gentamicin. Ren. Fail., 17: 21-26. 